\title{
肉兔配套系的选育研究进展
}

\author{
张翔宇, 黄邓萍, 谢晓红 \\ 四川省畜牧科学研究院, 成都 610066
}

摘要: 配套系在动物育种中能最大程度地利用品系的杂种优势, 以获得最大的经济效益, 配套系的选育是今后 肉兔遗传改良的发展趋势。肉免父系以选择平均日增重和上市体重为主, 断奶后生长性状一般与料肉比呈有利 的负遗传相关, 可作为料肉比的间接选择标准。而母系以选择断奶数和产仔数为主。大多数繁殖性状的遗传力 较低, 在进行遗传评估时应当尽可能全面地收集个体和相关亲缘关系个体的记录。重复观测值动物模型下的 BLUP 估计是进行肉兔专门化品系选育的主要方法。虽然窝内个体数性状的直接选择效率低于生长性状, 但其 杂种优势一般高于生长性状。通过计算杂交参数和比较同代杂种与纯种间的生产性能可以进行杂种优势的估 计。文章对肉兔专门化品系的选育、杂种优势的利用和良种繁育体系的建立进行了综述, 总结了肉兔专门化品 系的培育方法, 性状选择的标准以及杂种优势的估计结果。

关键词: 专门化品系; 肉兔; 杂种优势; 父系; 母系

\section{Progress on breeding for best-hybridized crossing of meat rabbits}

\section{ZHANG Xiang-Yu, HUANG Deng-Ping, XIE Xiao-Hong}

Sichuan Animal Science Academy, Chengdu 610066, China

\begin{abstract}
Best-hybridized crossing should ideally result in optimal exploitation of heterosis of lines and capitalize on expressed heterosis. Rabbit breeding is heading in the direction of breeding for best-hybridized crossing of meat rabbits. Most special sire lines are selected for post-weaning average daily gain and marketing weight. Post-weaning growth has a negative and favorable genetic correlation with the feed conversion ratio, which is used in indirect selection for feed conversion ratio. The most common selection criteria for special maternal lines are related to litter size at birth or at weaning. Since the heritability of most reproductive traits is low, we must collect as many individual and relative records as possible in the genetic evaluation of rabbits. The BLUP procedure under an animal repeatability model is the most common procedure used for evaluation of animals in selection programs for special lines of meat rabbits. Direct selection for litter size is less efficient than selection for post-weaning growth, but the estimation of heterosis is generally higher for litter size than that for the post-weaning growth. Evaluation of heterosis could be performed by estimating crossbreeding parameters in the
\end{abstract}

收稿日期: 2011-09-13; 修回日期: 2011-12-21

基金项目: 国家兔产业技术体系(编号：CARS-44-B-4), 四川省畜禽良种繁育专项(编号：SASA2009YZ005)和四川省畜禽育种攻关课题(编号： 2006YZGG-17)资助

作者简介: 张翔宇, 博士, 助理研究员, 研究方向 : 家兔育种。Tel: 028-84526166; E-mail: zhangxiangyu757@163.com

通讯作者: 谢晓红, 本科, 研究员, 研究方向 : 家兔育种与养殖。E-mail: xiexiaohongL@sina.com

致 谢: 感谢唐良美研究员在本文撰写过程中提出的宝贵意见和建议。

网络出版时间: 2012-3-16 14:50:48

URL: http://www.cnki.net/kcms/detail/11.1913.R.20120316.1450.002.html 
cross or comparing contemporary productivity among purebreds and crossbreds. Here, we reviewed breeding of special lines, exploitation of heterosis of crossbreds, and establishment of crossbreeding system of hybrid meat rabbits and summarized the methodologies of breeding special lines, criteria in selection programs, and the result of heterosis estimates.

Keywords: special lines; meat rabbits; heterosis; sire lines; maternal lines

提高肉兔生产水平, 增加经济效益是全球养兔 业共同关注的问题。遗传改良是提高肉兔生产水平, 获得良好社会经济效益和生态效益的重要手段 ${ }^{[1]}$ 。 肉兔主要经济性状的改良通常采用逐个改良的方法, 往往耗时太长。同时, 部分优良经济性状间存在拮 抗遗传相关性，导致这些性状无法同时获得遗传进 展 ${ }^{[2]}$ 。随着对大多数肉兔品种(品系)的经济性状的了 解和掌握, 利用杂种优势提高肉兔生产水平成为一 条改良肉兔重要性状的有效途径。杂交育种不仅能 够产生杂种优势，还能实现优良性状的聚集并缓解 近交衰退。过去肉兔的育种主要采用品系繁育, 群 体数量较小, 改良的目标性状单一, 短时间内能获 得较大的遗传进展, 但不利于多性状的选育。建立 在专门化品系杂交基础上的配套系能有效地利用杂 种优势, 提高生产性能, 满足市场需求, 弥补了单 一品系进行繁育的不足。肉兔配套系的建立主要包 括专门化品系选育和杂种优势测定两部分, 其最终 目的是充分利用杂种优势, 获得更大的遗传进展。

\section{1 专门化父系的选育}

经世界范围内肉兔育种研究人员的共同努力, 肉兔专门化父系在生长速度的遗传改良方面取得了 重大进展(表 1)。主要育种经验包括：以生长性状作 为专门化父系的选育方向和BLUP为其主要遗传评 估方法。在饲养过程中, 肉兔断奶后的饲料成本占 到了总生产成本的一半以上, 料肉比是影响肉兔产 业盈利的主要因素之一。但收集料肉比数据的成本 高昂, 有时会使用专门的电子设备记录个体的采食 量。因此, 在目前的肉兔繁育体系中, 料肉比的直接 选择已经不再是专门化父系的主要选择方式, 取而 代之的是以断奶重、上市体重和断奶后平均日增重 为选择标准的间接选择方法 ${ }^{[3,4]}$ 。前期的研究表明, 日增重主要受遗传效应调控, 遗传力中等偏高, 与 特定日龄个体重相比受窝效应的影响较小 [5]。同时, 日增重与料肉比呈有利的负遗传相关。因此, 日增 重是进行肉兔专门化品系培育时优先考虑的选择标 准。在进行肉兔专门化父系选育时, 通常利用生长

表 1 肉兔专门化品系的选育概况

\begin{tabular}{|c|c|c|c|c|c|}
\hline 品系 & 类型 & 来源 & 选择性状 & 选择方法 & 参考文献 \\
\hline line $\mathrm{R}$ & 专门化父系 & $\begin{array}{c}\text { 加利福尼亚兔和 } \\
\text { 一个合成父系 }\end{array}$ & $\begin{array}{c}\text { 4 11 周 } \\
\text { 平均日增重 }\end{array}$ & $\begin{array}{l}\text { 基于动物模型下 REML } \\
\text { 遗传估计的群体继代选育 }\end{array}$ & [8] \\
\hline line $\mathrm{C}$ & 专门化父系 & 新西兰白兔和加利福尼亚兔 & $\begin{array}{l}32 \sim 60 \text { 日龄 } \\
\text { 平均日增重 }\end{array}$ & $\begin{array}{l}\text { 基于动物模型下 BLUP } \\
\text { 遗传估计的群体继代选育 }\end{array}$ & [9] \\
\hline Line $\mathrm{S}$ & 专门化父系 & $\begin{array}{c}\text { 加利福尼亚兔、香槟兔和弗 } \\
\text { 朗德巨兔 }\end{array}$ & $\begin{array}{l}70 \text { 日龄 } \\
\text { 上市体重 }\end{array}$ & $\begin{array}{l}\text { 基于混合模型下 REML } \\
\text { 遗传估计的群体继代选育 }\end{array}$ & [10] \\
\hline line A & 专门化母系 & 新西兰白兔 & 断奶数 & $\begin{array}{l}\text { 以家系选择指数为基 } \\
\text { 础的群体继代选育 }\end{array}$ & [18] \\
\hline line $\mathrm{V}$ & 专门化母系 & 4 个专门化品系 & 断奶数 & $\begin{array}{l}\text { 利用重复观测值动物模型 } \\
\text { 进行 BLUP遗传估计的群体继代选育 } \\
\text { 利用重复观测值动物模型 }\end{array}$ & [18] \\
\hline line $\mathrm{P}$ & 专门化母系 & 2 个专门化母系 & 断奶数 & $\begin{array}{l}\text { 进行 BLUP 遗传估计的 } \\
\text { 不完全群体继代选育 }\end{array}$ & [19] \\
\hline Line LP & 专门化母系 & $\mathrm{H}$ 母系 & $\begin{array}{c}\text { 产仔数和 } \\
\text { 有效利用年限 }\end{array}$ & $\begin{array}{l}\text { 基于 BLUP 遗传估计 } \\
\text { 的群体继代选育 }\end{array}$ & [20] \\
\hline 齐兴肉兔 & 专门化母系 & 四川本地白兔和德国白兔 & 年产仔数 & $\begin{array}{l}\text { 基于 BLUP 遗传估计的 } \\
\text { 非完全闭锁群体继代选育 }\end{array}$ & {$[21]$} \\
\hline
\end{tabular}


性状对大群内的个体进行选择。这种方法可以节约 时间、人力和经费, 世代间隔也会大大缩短。BLUP 估计是进行肉兔专门化品系培育时常用的评估方法， 但渠化选择(Canalization)在近 10 年也开始用于肉兔 专门化父系的选育, 后者还能降低环境对遗传响应 的影响 ${ }^{[6]}$ 。就模型而言, 多性状模型对遗传参数(遗 传力、遗传相关和选择响应)造成有偏估计的可能性 大于重复观测值动物模型 ${ }^{[7]}$ 。西班牙培育的专门化 父系 $\mathrm{R}$ 系和 $\mathrm{C}$ 系已广泛应用于西班牙国内的肉兔生 产。R系源自两个融合的父系, 分别来自加利福尼亚 兔和 1981 年育成的终端父系。以 4 11 周的平均日 增重为选择标准, 利用动物模型下的限制性最大似 然估计进行选育 ${ }^{[8]}$ 。C系作为IRTA的终端父系, 来自 新西兰白兔和加利福尼亚兔的合成系。最初几个世 代以窝重为主要标准进行选择。之后开始以 32 60 日龄平均日增重为选择标准, 利用动物模型下的 BLUP进行群体继代选育 ${ }^{[9]}$ 。Lukefahr等 ${ }^{[10]}$ 利用加利 福尼亚兔、香槟兔和弗朗德巨兔组建合成系, 以 70 日龄上市体重为选择标准，采用混合模型下的 REML估计进行S系选育。

\section{2 专门化母系的选育}

繁殖性状(产仔数、产活仔数、断奶数等)的遗传 力一般较低, 因此肉兔专门化母系的培育相对专门 化父系的培育要复杂一些。在进行个体遗传评估时, 需要尽可能全面地收集个体本身和有亲缘关系个体 的繁殖记录。窝内个体数是影响利润函数的重要因 素, 因此产仔数和断奶数成为肉兔专门化母系的主 要选择标准(表 1) ${ }^{[11,12]}$ 。断奶重、断奶窝重和总泌乳 量一般作为提高母兔哺乳能力的选择标准 ${ }^{[13]}$ 。其他 的选择标准还包括：以提高活产仔数为目标的子宫 容量选择和以提高仔兔成活率为目标的个体初生重

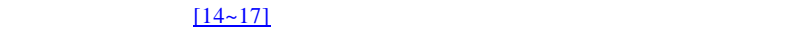
献较小，但选择模型中应该包括公兔效应。因此进 行母兔窝内个体数BLUP遗传估计时，一般采用动 物模型或重复观测值动物模型。就窝内个体数性状 而言, 利用上述两个模型估计的育种值基本相等。 肉兔专门化母系的选育见表 1 。 $A 、 V$ 和P兔是西班牙 培育的专门化母系, $\mathrm{A}$ 系源自新西兰白兔, 从第三世 代开始利用家系指数进行断奶数的选择。家系指数 包含个体、母本、全同胞和半同胞姐妹的断奶数
信息 ${ }^{[18]}$ 。V系在 4 个专门化品系合成的基础上, 利用 重复观测值动物模型下的BLUP估计个体育种值, 进行以断奶数为选择标准的群体继代选育 ${ }^{[18]}$ 。P系源 自两个专门化母系, 利用重复观测值动物模型的 BLUP估计育种值 ${ }^{[19]}$ 。西班牙的LP母系在 $\mathrm{H}$ 系的基础 上组建, 以产仔数和有效利用年限为选择标准, 利

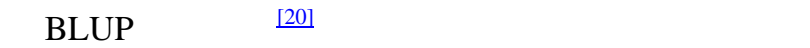

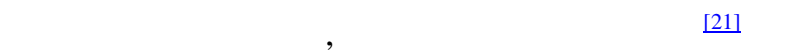
育种素材(本地白兔和引进兔种)种质性能测定和遗 传距离估计的基础上, 结合育种目标制定育种方案 [22 24]。采用家系内留种, 经 5 个世代的非完全闭锁 群体继代选育，最终育成年产仔数高、适应性强的 专门化母系, 后经四川省畜禽品种审定委员会正式 命名为“齐兴肉兔”。齐兴肉兔繁殖性状和生长性状的 遗传参数估计表明, 繁殖性状的遗传力偏低, 生长性 状间存在极显著遗传相关, 可利用早期生长性状进行 选择以加快选育速度 ${ }^{[25,26]}$ 。

\section{3 杂种优势利用}

大量的研究已经证实, 肉兔的生产性能在不同 的品种(品系)间差异显著 ${ }^{[27]}$ 。新西兰白兔、加利福 尼亚兔和比利时兔及其杂交后代的生长和屠宰性能 在各品种间差异显著。新西兰白兔的胴体性状较加 利福尼亚兔差, 加利福尼亚兔的屠宰体重较轻, 而 屠宰率较高 ${ }^{[28]}$ 。Lukefahr等 ${ }^{[29]}$ 报道了新西兰白兔的 日增重和上市体重低于比利时兔。加利福尼亚兔的 初配体重显著低于比利时兔和新西兰白兔, 比利时 兔的初配体重大于新西兰白兔 ${ }^{[30]}$ 。肉兔品种(品系) 间生产性能的差异是获得杂种优势的重要前提。

杂种优势的利用是建立配套系的最终目的，但 杂种优势的作用机理目前尚不完全清楚。一般而言, 杂种优势的大小取决于群体间基因频率差异的大小, 并且当群体内的等位基因频率相对固定时杂种优势 达最大, 即品种(品系)内性状的差异要小, 品种(品 系)间性状的差异要大 ${ }^{[31]}$, 繁殖性状较生长性状更易 获得杂种优势。估计杂种优势的常用方法有两种： 一种方法是进行杂交参数的估计, 该方法对统计模 型和计算方法要求较高; 另一种方法是通过对同代 杂交兔和纯种兔生产性能的比较进行杂种优势的估 计。这种方法对统计和算法要求相对较低, 国内相 关研究大多采用该法。西班牙 3 个专门化母系(A、 
$\mathrm{V}$ 和P)与 2 个专门化父系 $(\mathrm{R}$ 和 $\mathrm{C}$ )进行双列杂交, 采用 动物模型下的贝叶斯算法估计生长性状和料肉比的 杂交参数, 结果表明杂交后代的生长性状和料肉比 保留了父系的特点 ${ }^{[32]}$ 。Piles等 ${ }^{[33]}$ 通过 A、V 和P 3 个 专门化母系间的双列杂交, 估计了母兔有效利用年 限的杂交参数, 结果表明 AP和VP组合的杂种优势 显著。Nofal等 ${ }^{[34]}$ 观测到断奶性状的杂种优势变化范 围为 $10 \%$ 17.7\%, 而Prayaga 等 ${ }^{[27]}$ 观测到断奶性状 的杂种优势变化范围为 $14.6 \%$ 41.9\%。加利福尼亚 兔和新西兰白兔的杂交后代在断奶数上存在杂种优 势, 其断奶数高于纯种的亲本 ${ }^{[35]}$ 。Partridge等 ${ }^{[36]}$ 发现 配怀率存在杂种优势。以德国巨型兔为父本的杂交 后代在生长速度、饲料利用率和屠宰性能上存在明 显的杂种优势 [37]。哈尔滨白兔是我国自主培育的大 型肉兔品种, 以其为父本的杂交后代体重、饲料报 酬和屠宰率存在杂种优势 [38]。有研究指出, 以中国 白兔为父本分别与新西兰白兔和加利福尼亚兔杂交, 产仔数的杂种优势率分别为 $9.59 \%$ 和 $9.17 \%$ [39]。加利 福尼亚公兔与哈白母兔杂交的母兔再与新西兰公兔 杂交, 断奶成活数、35 日龄断奶数的杂种优势率分 别为 $14.20 \%$ 和 $31.26 \%$ [40]。齐兴肉兔分别与齐卡巨 型白兔、齐卡大型新西兰白兔、哈尔滨白兔和比利 时兔杂交, 产仔数和初生窝重均表现出杂种优势
[41]。加利福尼亚兔无论作杂交父本还是母本, 杂交 后代肉质性状的杂种优势明显 ${ }^{[42]}$ 。

\section{4 配套繁育体系的建立}

以获取最大杂种优势兼顾市场需求为原则，根 据杂种优势估计结果, 确定各系在杂交体系中的位 置和最佳的制种模式，建立配套繁育体系。该体系 包括：育种场、祖代场、父母代场和商品代场。育 种场主要进行专门化品系的选育, 祖代场主要进行 祖代的纯繁, 而父母代场主要是进行父母代的制 种。商品代场则是商品代肉兔肥育的场所。这种以 育种场为核心，繁殖场为中介，商品代场为目的的 肉兔生产“金字塔”结构, 能迅速地将遗传改良成果 转化成生产力, 充分利用杂种优势, 生产高品质的 商品肉兔。目前, 比较著名的肉兔配套系有: 法国 的伊普吕(HYPLUS)配套系和伊拉(HYLA)配套系, 德国的齐卡(ZIKA)配套系 ${ }^{[43]}$ 。肉兔配套系一般由 3 个或 4 个专门化品系组成, 采取三元杂交或双杂交 (表 2)。伊拉肉兔由A、B、C、D 4 个系组成。A、B 系杂交产生父母代父本，C、D系杂交产生父母代母 本 ${ }^{[44]}$ 。齐卡配套系包括 3 个品系 :齐卡巨型白兔 $(G)$ 、 齐卡大型新西兰 $(N)$ 、齐卡白兔 $(\mathrm{Z}){ }^{[45]}$ 。伊普吕肉兔 的父母代亲本共有 4 个品系, 商品兔生产一般

表 2 世界著名的肉兔配套系

名称 育成国家 杂交方式


采用四系配套 ${ }^{[46,47]}$ 。利用配套系生产的商品肉兔具 有增重快、屠宰率高和饲料利用率高的特点。

\section{5 结 语}

配套系的建立是一项系统工程, 专门化品系繁 育和杂种优势测定各环节紧密相扣。优质商品代肉 兔是良种繁育体系的最终产品。必须以市场需求为 目标, 以广泛的种质资源为基础, 以现代育种理论 为依据，采用品种内分化和品系间合成相结合的方 法进行专门化品系选育, 在杂种优势测定结果的基 础上，确定最佳制种模式，建立配套繁育(制种)体 系。需要指出的是将分子生物学技术应用于肉兔专 门化品系的选育和杂种优势的预测将是肉兔配套系 选育的发展趋势。就目前而言，家兔分子标记的研 究主要集中在遗传多样性分析和亲子鉴定方面 ${ }^{[48]}$ 。 虽然短期内无法实现将标记辅助选择(MAS)广泛应 用于家兔育种，但已有家兔功能基因多态性与生产 性状相关性的报道 ${ }^{[49 ~ 51]}$, 这将是开展肉兔标记辅助 选择的重要基础。配套系建立后还应根据市场需求 培育新的品系和改变杂交模式, 建立动态育种机制, 不断建立新的配套系，提高经济效益。

\section{参考文献(References):}

[1] McNitt JI, Lukefahr SD. Breed and environmental effects on postweaning growth of rabbits. J Anim Sci, 1993, 71(8): 1996-2005.DOI

[2] Larzul C, Gondret F, Combes S, de Rochambeau H. Divergent selection on 63-day body weight in the rabbit: response on growth, carcass and muscle traits. Genet Sel Evol, 2005, 37(1): 105-122. DOI

[3] Moura ASAMT, Costa ARC, Polastre R. Variance components and response to selection for reproductive, litter and growth traits through a multi-purpose index. World Rabbit Sci, 2001, 9(2): 77-86.

[4] Prayaga KC, Eady SJ. Rabbit farming for meat production in Australia: Preliminary estimates of economic values for production traits. Asian-Australas J Anim Sci, 2000, 13(Suppl.): 357-359. DOI

[5] Krogmeier D, Dzapo V, Mao IL. Additive genetic and maternal effects on postweaning growth and carcass traits in rabbits. J Anim Breed Genet, 1994, 111(1-6): 289-297. DOI

[6] Garreau H, Bolet G, Larzul C, Robert-Granié C, Saleil G, SanCristobal M, Bodin L. Results of four generations of a canalising selection for rabbit birth weight. Livest Sci, 2008, 119(1-3): 55-62. DOI

[7] Piles M, García ML, Rafel O, Ramon J, Baselga M. Genetics of litter size in three maternal lines of rabbits: Repeatability versus multiple-trait models. J Anim Sci, 2006, 84(9): 2309-2315. DOI

[8] Estany J, Camacho J, Baselga M, Blasco A. Selection response of growth rate in rabbits for meat production. Genet Sel Evol, 1992, 24(6): 527-537. DOI

[9] Gómez EA, Rafel O, Ramon J. Preliminary genetic analyses of Caldes line: A selection experiment for a global objective. In: Proceeding of the 7th World Rabbit Congress. Valencia, Spain, 2000: 417-424. DOI

[10] Lukefahr SD, Odi HB, Atakora JK. Mass selection for 70-day body weight in rabbits. J Anim Sci, 1996, 74(7): 1481-1489. DOI

[11] Quevedo F, Cervera C, Blas E, Baselga M, Costa C, Pascual JJ. Effect of selection for litter size and feeding programme on the performance of young rabbit females during rearing and first pregnancy. Anim Sci, 2005, 80(2): 161-168. DOI

[12] García ML, Baselga M. Estimation of genetic response to selection in litter size of rabbits using a cryopreserved control population. Livest Prod Sci, 2002, 74(1): 45-53. DOI

[13] Al-Saef AM, Khalil MH, Al-Homidan AH, Al-Dobaib SN, Al-Sobayil KA, García ML, Baselga M. Crossbreeding effects for litter and lactation traits in a Saudi project to develop new lines of rabbits suitable for hot climates. Livest Sci, 2008, 118(3): 238-246. DOI

[14] Blasco A, Ortega JA, Climent A, Santacreu MA. Divergent selection for uterine capacity in rabbits. I. Genetic parameters and response to selection. J Anim Sci, 2005, 83 (10): 2297-2302. DOI

[15] Mocé ML, Santacreu MA, Climent A, Blasco A. Divergent selection for uterine capacity in rabbits. III. Responses in uterine capacity and its components estimated with a cryopreserved control population. J Anim Sci, 2005, 83 (10): 2308-2312. DOI

[16] Santacreu MA, Mocé ML, Climent A, Blasco A. Divergent selection for uterine capacity in rabbits. II. Correlated response on litter size and its components estimated with a cryopreserved control population. J Anim Sci, 2005, 83 (10): 2303-2307. DOI

[17] Bolet G, Garreau H, Joly T, Theau-Clément M, Falieres J, Hurtaud J, Bodin L. Genetic homogenisation of birth weight in rabbits: Indirect selection response for uterine horn characteristics. Livest Sci, 2007, 111(1-2): 28-32. DOI 
[18] Estany J, Baselga M, Blasco A, Camacho J. Mixed model methodology for the estimation of genetic response to selection in litter size of rabbits. Livest Prod Sci, 1989, 21(1): 67-75. DOI

[19] Gómez EA, Rafel O, Ramon J, Baselga M. A genetic study of a line selected on litter size at weaning. In: Proceeding of the 6th World Rabbit Congress. Toulouse, France, 1996: 289-292.

[20] Sánchez JP, Theilgaard C, Mínguez C, Baselga M. Constitution and evaluation of a long-lived productive rabbit line. J Anim Sci, 2008, 86(3): 515-525. DOI

[21] 唐良美, 谢晓红, 刘曼丽, 黄邓萍, 龙继蓉, 兰永亿. 肉 兔专门化新品系 $\mathrm{A}$ 兔的培育与利用研究初报. 四川畜 牧兽医, 1999, 26(S1): 30-32. DOI

[22] 刘曼丽, 唐良美, 颜洁邦, 张继国, 谢小红, 黄邓萍. 几 个主要引进肉兔品种的生产性能测定. 四川畜牧兽医, 1989, 16(1): 5-8. DOI

[23] 冯蜀举, 郭春华, 刘曼丽, 钟勇. 中国白兔与其它家兔 品种间遗传关系的研究. 西南民族大学学报 (自然科学 版), 1992, 18(3): 314-318. DOI

[24] 刘曼丽, 唐良美, 谢小红. 四川白兔部分种质特征和生 产性能测定．四川畜牧兽医, 1992, 19(1): 11-13. DOI

[25] 龙继蓉, 蒋必光, 谢晓红, 唐良美, 黄邓萍, 雷峮, 张杰, 向俊琳, 李华. A系兔生长性状遗传参数的估测. 四川农 业大学学报, 2000, 18(1): 62-64. DOI

[26] 龙继蓉, 蒋必光, 谢晓红, 唐良美, 黄邓萍, 雷岷, 张杰, 向俊琳, 李华. A系兔繁殖性状遗传参数的估测. 四川农 业大学学报, 2000, 18(2): 179-182. DOI

[27] Prayaga KC, Eady SJ. Performance of purebred and crossbred rabbits in Australia: doe reproductive and pre-weaning litter traits. Aust J Agric Res, 2002, 53(9): 993-1001. DOI

[28] Prayaga KC, Eady SJ. Performance of purebred and crossbred rabbits in Australia: Individual growth and slaughter traits. Aust J Agric Res, 2003, 54(2): 159-166. DOI

[29] Lukefahr S, Hohenboken WD, Cheeke PR, Patton NM. Genetic effects on maternal performance and litter pre-weaning and post-weaning traits in rabbits. Anim Prod, 1984, 38(2): 293-300. DOI

[30] Lukefahr S, Hohenboken WD, Cheeke PR, Patton NM. Doe reproduction and preweaning litter performance of straightbred and crossbred rabbits. J Anim Sci, 1983, 57(5): 1090-1099. DOI

[31] Falconer DS. Introduction to Quantitative Genetics, 2nd ed. London: Longmans Green, 1981. DOI

[32] Orengo J, Piles M, Rafel O, Ramon J, Gómez EA. Crossbreeding parameters for growth and feed consumption traits from a five diallel mating scheme in rabbits. J Anim Sci, 2009, 87(6): 1896-1905. DOI
[33] Piles M, Sánchez JP, Orengo J, Rafel O, Ramon J, Baselga M. Crossbreeding parameter estimation for functional longevity in rabbits using survival analysis methodology. $J$ Anim Sci, 2006, 84(1): 58-62. DOI

[34] Nofal RY, Toth S. Evaluation of seven genetic groups of rabbits for some reproductive traits in the first three parities. Arch Tierzucht, 1996, 39(6): 623-631.

[35] Afifi EA, Khalil MH, Khadr AF, Youssef YMK. Heterosis, maternal and direct effects for postweaning growth traits and carcass performance in rabbit crosses. J Anim Breed Genet, 1994, 111(1-6): 138-147. DOI

[36] Partridge GG, Foley S, Corrigall W. Reproductive performance in purebred and crossbred commercial rabbits. Anim Prod, 1981, 32(3): 325-331. DOI

[37] 李华, 蒋必光, 王康宁. 肉兔双列杂交遗传效应的分析. 中国养兔杂志, 1995, (4): 30-34. DOI

[38] 朱瑾佳, 程雷, 张克敏, 王晓节, 戴德芬. 哈尔滨白兔 产肉性能和杂交试验. 中国畜牧杂志，1989，25(5)：2122. DOI

[39] 程园, 吴运德, 胡迪先, 马万能, 刘秀英, 肖超能, 朱小 䑣, 张立. 商品肉兔杂交组合篮选试验. 中国养兔杂志, 1998, (6): 13-16. DOI

[40] 张恒业, 周大生, 朱宽佑, 张焕, 林锋. 肉兔强度育肥 及杂交性能试验研究. 中国养兔杂志, 1997, (3): 17-21. DOI

[41] 黄邓萍, 唐良美, 刘曼丽, 谢晓红, 陈文昭, 兰永忆, 刘 林. 四个大型肉兔品种(系)作父本的杂交组合试验. 四 川畜牧兽医, 1995, 22(2): 17-18. DOI

[42] 李华, 于辉, 蒋必光, 郑维明. 兔肉品质配合力与杂种 优势的研究. 中国养兔, 2001, (2): 22-26. DOI

[43] 沈幼章. 世界养兔生产及兔业技术进展简况. 中国养兔, 2001, (2): 32-34. DOI

[44] 谭启兆. 曾祖代伊拉(Hyla)配套系落户“绿洲”. 中国养 兔, 2001, 1(1): 44. DOI

[45] 刘曼丽, 唐良美, 兰永忆, 谢晓红, 陈文昭, 刘林. 齐卡 配套系肉兔在四川的选育. 西南农业学报, 1994，7(2): 89-94. DOI

[46] Vostrý L, Mach K, Jakubec V, Dokoupilová A, Majzlík I. The influence of weaning weight on growth of the hyplus broiler rabbit. In: Proceeding of the 9th World Rabbit Congress. Verona, Italy, 2008: 255-259. DOI

[47] Hypharm. 2011. http://www.hypharm.fr/media/doc/fiche_ multi.pdf. DOI

[48] 韩春梅, 张嘉保, 高庆华, 陈庆波. 微卫星DNA在吉戎 兔亲子鉴定中的应用研究. 遗传, 2005, 27(6): 903-907. DOI

[49] 李春笑, 蒋美山, 陈仕毅, 赖松家. 兔成纤维细胞生长 因子 5(FGF5)基因SNP及其与产毛量的相关分析. 遗传, 2008, 30(7): 893-899. DOI 
[50] 蒋美山, 陈仕毅, 赖松家, 邓小松, 陈云, 万洁. 兔黑素 皮质素受体 4(MC4R)基因多态性及其与体重及屠体性 状的关联研究. 遗传, 2008, 30(12): 1574-1578. DOI

[51] 邓小松, 万洁, 陈仕毅, 王彦, 赖松家, 蒋美山, 徐敏. 兔GHR基因单核苷酸多态性及其与屠宰性状的相关性. 遗传, 2008, 30(11): 1427-1432. DOI 